\title{
Vacuum Circuit Breaker Postarc Current Modelling Based on the Theory of Langmuir Probes
}

\author{
Ezra P. A. van Lanen, Member, IEEE, René Peter Paul Smeets, Senior Member, IEEE, \\ Marjan Popov, Senior Member, IEEE, and Lou van der Sluis, Senior Member, IEEE
}

\begin{abstract}
High-resolution measurements on the postarc current in vacuum circuit breakers (VCBs) reveal a period, immediately following current-zero, in which the voltage remains practically zero. The most widely used model for simulating the interaction between the postarc current with the electrical circuit lacks a proper explanation for this event, and hence, it needs to be complemented. We demonstrate that the breaker's electrical behavior during this zero-voltage period can be explained by using the theory of a Langmuir probe. Such probes are used to investigate plasma properties such as the ion density and the electron temperature, and we extrapolate its theory to the VCB. After the voltage-zero period, when the transient recovery voltage starts to rise, the breaker's electrical behavior is mainly determined by the expansion of an ionic space-charge sheath in front of the cathode. In addition to the current from the Langmuir probe model, the time change of the electric field inside the sheath gives a displacement current. Instead of solving the complicated plasma equations to find the displacement current, we use an approximation by simulating it with the aid of a voltage-dependent sheath capacitance. We programmed the model as a function block in Matlab's SimPowerSystems to facilitate its application in different electrical circuits.
\end{abstract}

Index Terms-Postarc current, postarc modeling, short-circuit current, vacuum circuit breaker (VCB).

\section{INTRODUCTION}

$\mathbf{V}$ ACUUM CIRCUIT breakers (VCBs) are devices in electrical power systems that interrupt short-circuit currents. They are most widely used in distribution systems with voltage levels up to $72.5 \mathrm{kV}$ [1], [2]. The current-interruption process in a VCB is done by a metal-vapor arc, which is more commonly known as a vacuum arc [3]. This arc appears as soon as the breaker's contacts separate, and it continues to exist until its energy input ceases. In ac circuits, such a moment occurs always at the power-frequency current zero.

The actual current interruption in a VCB occurs by means of arc extinction. Therefore, a thorough understanding of the arc is imperative for the development of new breakers. However, since direct measurement on the arc is difficult because, to maintain vacuum, these breakers are sealed for life; the only way information can be obtained is by indirect research. This

Manuscript received May 4, 2006; revised January 18, 2007.

E. P. A. van Lanen, M. Popov, and L. van der Sluis are with the Electrical Power Systems Laboratory, Faculty of Electrical Engineering, Mathematics and Computer Science, Delft University of Technology, 2628 CD Delft, The Netherlands (e-mail: e.p.a.vanlanen@tudelft.nl).

R. P. P. Smeets is with the Faculty of Electrical Engineering, Eindhoven University, 5600 MB Eindhoven, The Netherlands, and also with the KEMA Transmission and Distribution Testing, 6812 AR Arnhem, The Netherlands.

Digital Object Identifier 10.1109/TPS.2007.895210 entails investigating the performance of a VCB by measuring its electrical behavior.

When the arc extinguishes, it leaves a conductive plasma behind, making an electrical feature possible, which is called the postarc current. In VCBs, the characteristics of postarc currents are particularly distinctive, and since it might indicate the performance of the breaker, it has been investigated thoroughly in previous studies [4]-[9].

Various models have been developed in the past for the simulation of the postarc processes that take place inside a VCB. Almost all of these models use the principle of the movement of charged particles under the influence of a transient recovery voltage (TRV), expressed in simplified equations [10]-[12]. With such a model, the postarc-circuit interaction can be simulated, and to some extent, the properties of the postarc plasma can be estimated, e.g., the initial ion density and its rate of decay.

Although the most widely used postarc current model (Andrews and Varey [13], [14]) simulates the electrical postarc behavior rather well, it has some limitations about its initial assumptions, one of which is explained in Section II. Moreover, it is our experience that this model requires special treatment to make it numerically stable, which complicates its application.

The previously stated problems lead to the conclusion that the postarc current model needs to be revised. Since we are mainly interested in the interaction of the breaker with the circuit, we try to avoid the complicated physics of plasma-sheath dynamics, and hence their considerable numerical efforts to solve the plasma equations, of which numerous examples can be found in the literature [15]-[17]. To summarize, the model should meet the following requirements:

1) numerically stable;

2) easy to solve;

3) easy to apply in any electrical circuit;

4) simulate the vacuum postarc's electrical behavior;

5) require a short list of parameters.

Section II describes the problem of the existing postarc current model. Section III gives a solution for this. Section IV presents a way to model the postarc current accordingly. Section V discusses the validity of the model, and Section VI draws conclusions.

\section{Voltage-Zero Period}

Fig. 1 shows a typical VCB postarc current. Details about the test circuit in which the results were obtained and the measuring equipment with which the data were recorded can be 


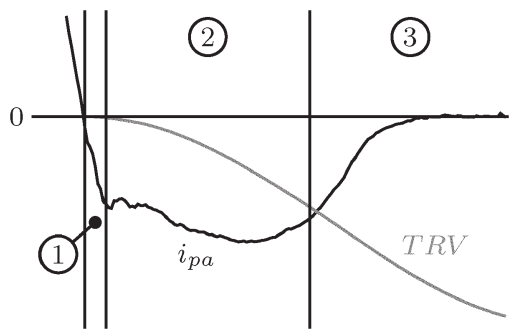

Fig. 1. Postarc current in a VCB. The numbers refer to the phases as explained in the text.

found in [14], [18], and [19]. For the usual explanation of this phenomenon, the postarc current is divided into three phases, and for clarity, we describe these in the following.

During arcing, ions are launched from the cathode toward the anode. At current zero, the ions that are still present between the contacts continue to move toward the anode as a result of their inertia. Electrons are much lighter than ions, and it is assumed that they adapt their speed immediately to a change in the electric field. As a result, the electrons match their velocity with the ion velocity to compensate for the ion current at current zero.

We now enter phase 1 . Immediately after current zero, the electrons reduce their velocity, and a net flux of positive charge arrives at the postarc cathode. This process continues until the electrons reverse their direction, and until this moment, the net charge inside the gap is zero. With no charge, we know from Poisson's equation that the voltage in this phase is zero.

As soon as the electrons reverse their direction, the postarc current enters its second phase. In this phase, the electrons move away from the cathode, leaving an ionic space-charge sheath behind. Now, the gap between the electrodes is not neutral anymore, and the circuit forces a TRV across it. This voltage is almost completely taken up by the sheath, which, contrary to the plasma, is not neutral.

The sheath continues to expand into the interelectrode gap until it reaches the new anode. At that moment, the postarc current reaches its third phase. The electrical current ceases, since all electrons have been removed from the gap. The process that determines the breaker's electrical behavior in this phase is explained in [20].

Some phenomena can be explained with this model, but the voltage zero in the first phase remains a problem. If ions and electrons are indeed moving toward the postarc cathode in this phase, they leave behind an empty gap between the plasma and the new anode. By definition of the model, charge sources are absent after current zero; hence, no charge leaves the postarc anode to fill up this gap. This implies breaking the electrical connection between the plasma and the anode, and hence, a voltage should rise across the gap during this phase. However, our measurement, such as the one in Fig. 2, clearly shows a voltagezero phase immediately following current zero. Apparently, this model should be complemented with an additional theory.

\section{LAngmuir Probe Theory Applied to the VCB}

The voltage-zero period described in Section II can be explained when we take the thermal velocity of the particles into
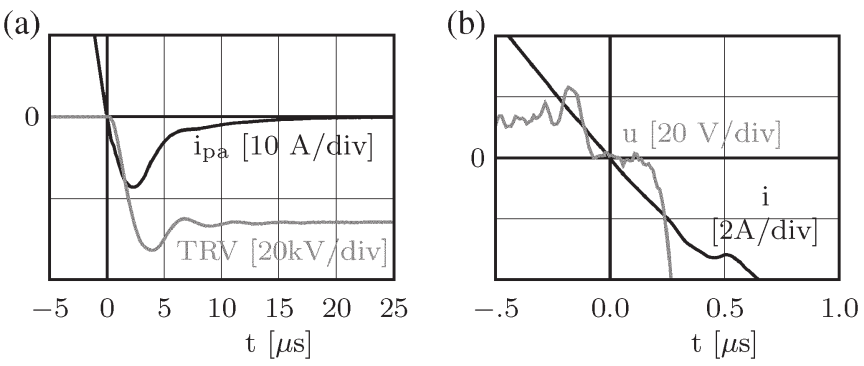

Fig. 2. Typical example of the voltage-zero phase.

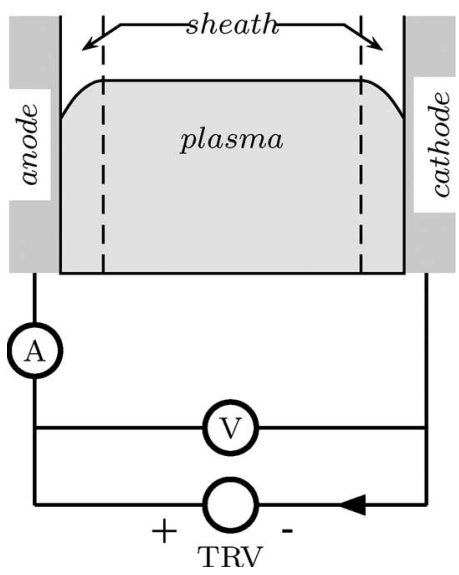

Fig. 3. Configuration of the postarc current model.

account. In that case, the pressure of electrons and ions causes the plasma to remain neutral in the voltage-zero phase. The breaker's electrical behavior then resembles that of a Langmuir probe, of which the relation between the current and voltage is known [21]-[23]. This facilitates the description for the voltage-zero period. For the application of this theory to the $\mathrm{VCB}$, we start with a series of assumptions, some of which do not immediately apply to the actual situation.

First, consider the configuration of Fig. 3. The electrodes are large and placed closely together, so we assume that the interelectrode properties only change in the direction perpendicular to them. This limits the problem to one dimension. We assume further that the plasma is stationary and equally distributed along the gap, and that all particles reaching an electrode are absorbed by it. The electron temperature is higher than the ion temperature, no collisions take place between particles in either the plasma or the sheaths, and finally, no new plasma is generated. The process of events following current zero is illustrated in Fig. 4.

At $t=0$, both the voltage across the gap and the current through the circuit are zero. Since their thermal energy is higher and their mass is lower, electrons have a higher thermal velocity than ions. Because of this, the flux of electrons at an electrode would be larger than the flux of ions, which results in an electrical current. However, since both electrodes experience the same flux in opposite direction, the current remains zero. An electric field in front of the electrodes repels the surplus of electrons to maintain a net charge flux of zero. This means that there is a potential difference between the plasma and the 
(a)

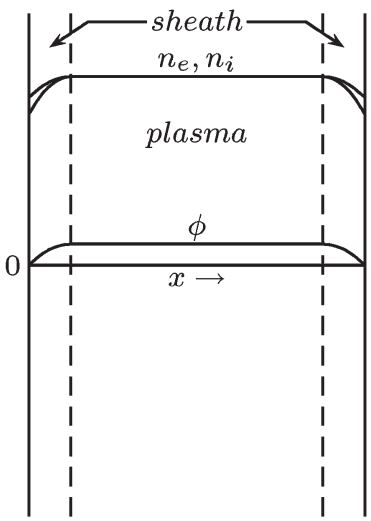

(b)

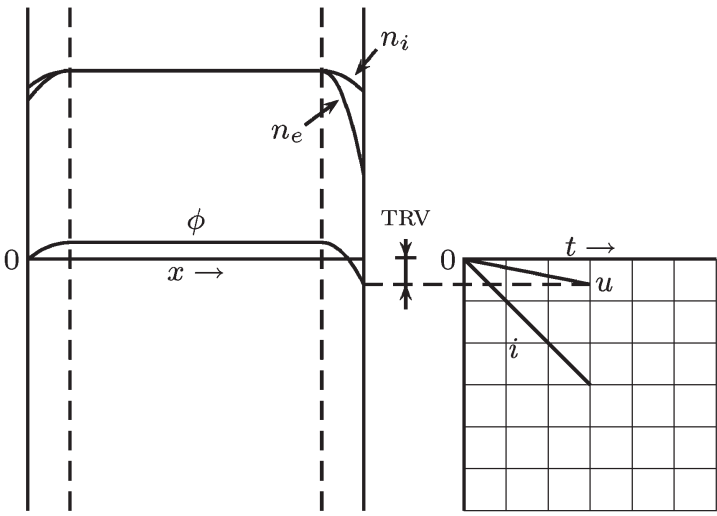

(c)

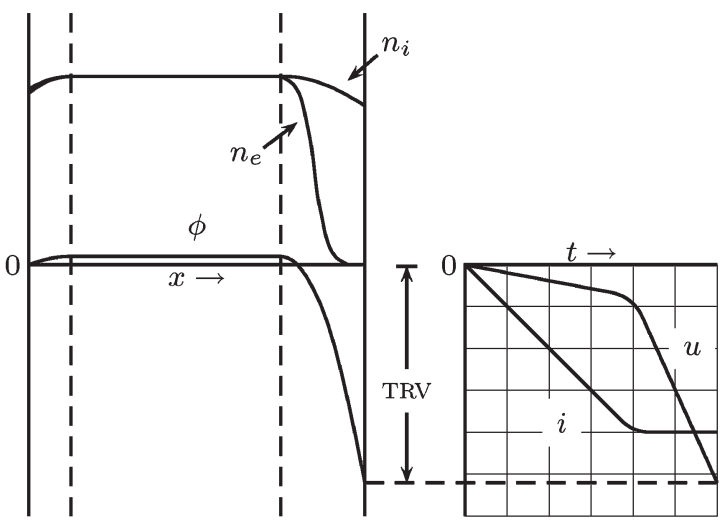

Fig. 4. Illustrative example of the events following current zero. $n_{\mathrm{i}}$ and $n_{\mathrm{e}}$ are the ion density and the electron density, respectively, and $\phi$ is the potential inside the gap. (a) Situation at $t=0$. (b) Situation during the low-voltage period. (c) Situation after ion-current saturation.

electrodes, which is mainly distributed across a small ionic space-charge sheath in front of the electrodes [see Fig. 4(a)].

When the TRV starts to rise, the electric field in front of the cathode increases and repels more electrons. However, due to their temperature, a large amount of electrons can cross the electric field to reach the cathode. The change of the flux of charged particles at the contacts makes an electrical current possible, but because an abundance of electrons is still present in the cathodic sheath, the gap is still quasi-neutral, and as a result of Poisson's equation, the voltage across the gap remains low Fig. 4(b).

As the TRV continues to increase, the electric field in front of the cathode repels more electrons, until eventually, it becomes too strong for any electron to reach the cathode. At this point, the current is limited to the flux of ions reaching the cathode Fig. 4(c), and an increase in voltage would only lead to an increase of the cathodic sheath width in front of the cathode, whereas the ion saturation current remains constant.

To find the relation between the current and the voltage for the VCB in the voltage-zero period, we start by investigating the interaction between the plasma and one contact.

For the stability of the sheath, the electric field in front of a contact partly penetrates the quasi-neutral plasma. This field not only starts the deceleration of electrons, but it also accelerates ions into the direction of the sheath to velocities exceeding their thermal velocity. As a result, ions enter the sheath with Bohm velocity $v_{\mathrm{B}}$. Without further explanation on how $v_{\mathrm{B}}$ is derived, we present its expression as [21]-[23]

$$
v_{\mathrm{B}}=\left(\frac{k T_{\mathrm{e}}}{m_{\mathrm{i}}}\right)^{1 / 2}
$$

where $k$ is the Boltzmann's constant, $T_{\mathrm{e}}$ is the electron temperature, and $m_{\mathrm{i}}$ is the ion mass. With $v_{\mathrm{B}}$, we can evaluate the ion saturation current as

$$
i_{\mathrm{i}, \mathrm{sat}}=q_{\mathrm{i}} v_{\mathrm{B}} n_{\mathrm{i}} A
$$

where $q_{\mathrm{i}}$ is the average ion charge, $n_{\mathrm{i}}$ is the ion density at the plasma-sheath boundary, and $A$ is the contact's surface area.

When a Maxwellian distribution function is assumed for electrons at the plasma-sheath boundary, the electron current $i_{\mathrm{e}}$ is found as

$$
i_{\mathrm{e}}=J_{\mathrm{e}, \mathrm{sat}} A \exp \left(\frac{q_{\mathrm{i}} V_{\mathrm{p}}}{k T_{\mathrm{e}}}\right)
$$

where $J_{\mathrm{e}, \mathrm{sat}}$ is the electron saturation current density, and $V_{\mathrm{p}}$ is the voltage drop across the sheath. Similar to the ion saturation current, the electron saturation current is reached when a contact's potential becomes positive enough with respect to the plasma. In absolute values, it is much higher than the ion saturation current.

In the configuration of Fig. 3, the current $i$ through the loop is equal at both contacts, hence

$$
i=i_{\mathrm{e}}^{a}-i_{\mathrm{i}, \mathrm{sat}}^{a}=i_{\mathrm{i}, \mathrm{sat}}^{c}-i_{\mathrm{e}}^{c}
$$

where the superscripts $a$ and $c$ refer to the anode and cathode, respectively. When we substitute $i_{\mathrm{e}}$ in (4) with (3) and use $V=$ $V_{\mathrm{p}}^{a}-V_{\mathrm{p}}^{c}$ for the voltage across the gap, we obtain

$$
\frac{i+i_{\mathrm{i}, \mathrm{sat}}^{a}}{i_{\mathrm{i}, \mathrm{sat}}^{c}-i}=\frac{A^{a}}{A^{c}} \exp \left(\frac{q_{\mathrm{i}} V}{k T_{\mathrm{e}}}\right) .
$$

We assumed that the contacts have equal size; hence, (5) can be simplified to

$$
i=i_{\mathrm{i}, \mathrm{sat}} \tanh \left(\frac{q_{\mathrm{i}} V}{2 k T_{\mathrm{e}}}\right) .
$$

Equation (6) indicates that the total current is restricted to the ion saturation current at either one of the contacts. Hence, 
(a)

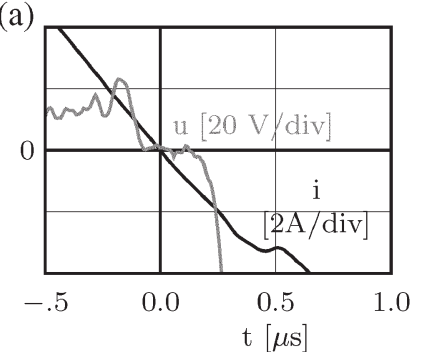

(b)

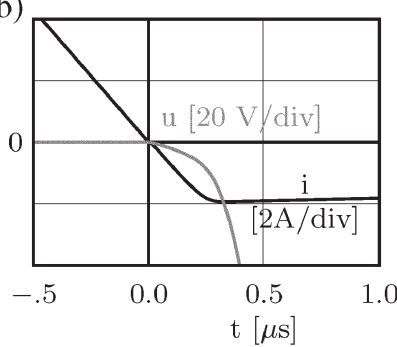

Fig. 5. Simulation of the low-voltage period following current zero. (a) Measured data. (b) Simulation results.

neither contact will experience the much higher electron saturation current in this model.

Next, we compare this model with the measurement of Fig. 2. In this particular example, the test object was subjected to a short-circuit current that largely exceeded the breaker's rated value. As such, the amount of residual charge at current zero is relatively large, making it easier to observe the low-voltage phase. The model is simulated in a circuit that is similar to the one from which the measurement has been obtained.

The quantities $T_{\mathrm{e}}, m_{\mathrm{i}}$, and $n_{\mathrm{i}}(0)$ (the initial ion density) cannot be measured with our measuring equipment, and their value has to be estimated or taken from the literature. $T_{\mathrm{e}}$ is normally in the order of 2-10 eV [24]-[27], and we assume that only copper ions are present inside the gap, so $m_{\mathrm{i}}=$ $1.06 \cdot 10^{-25} \mathrm{~kg}$. We assume further that the ion density decays exponentially $\left(n_{\mathrm{i}}=n_{\mathrm{i}}(0) \exp (-t / \tau)\right)$.

The results from measurements on the initial ion density and its decay have also been reported frequently in the literature, with values for $n_{\mathrm{i}}(0)$ in the range of $10^{17}-10^{19} \mathrm{~m}^{-3}$ and values for $\tau$ ranging from 0.5 to $84 \mu \mathrm{s}$ [6], [7], [9], [28], [29]. We take values within these ranges to match the model's results with the measured data.

The contact diameter of the VCB that we used in our investigation is $4 \mathrm{~cm}$, and hence, $A$ is $12.5 \mathrm{~cm}^{2}$. We assume for the simulation that $T_{\mathrm{e}}, q_{\mathrm{i}}$, and $n_{\mathrm{i}}(0)$ are $3 \mathrm{eV}, 1.8 \cdot 1.6 \cdot 10^{-19} \mathrm{C}$, and $2.8 \cdot 10^{18} \mathrm{~m}^{-3}$, respectively, and that the time constant $\tau$ for the ion decay is $20 \mu \mathrm{s}$.

Fig. 5 presents the results, and we observe indeed a voltagezero period in the simulation that resembles the one in the measurement.

The rise of the recovery voltage entails a fast time-change in the electric field inside the ionic space-charge sheath in front of the cathode. This yields a displacement current, which has not yet been taken into account in our model so far. Hence, in the model, the current remains equal to the saturation current, whereas the measured current continues to increase for some time.

\section{Postarc Current Model}

As explained in Section III, the voltage-zero model needs complementing, in order to take the sheath growth in front of the cathode into account. With regard to the model requirements stated in Section I, instead of modeling the complicated physical plasma-sheath processes, we use a deliberate pragmatic approach for this purpose.
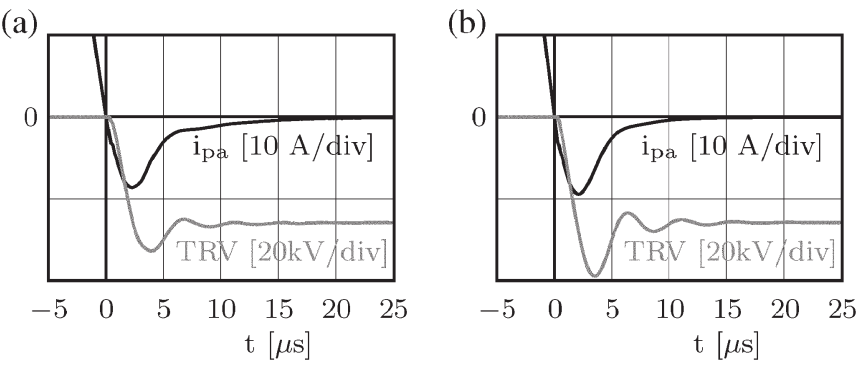

Fig. 6. Simulation of the postarc current. (a) Measured data. (b) Simulation results.

Contrary to the assumption in Section III, we take into account the finite size of the electrodes for the modeling of the sheath expansion but continue to approach the problem in one dimension. As the sheath grows in all directions, the plasma-sheath boundary grows, which effectively increases the cathode's area. As a result, more ions are collected at the sheath boundary, and the ion-saturation current increases. (The actual processes involved with the sheath expansion are more complicated than this, but for our model, this explanation suffices.)

For simplicity, we assume that the effective cathode area increases linearly with the voltage; thus

$$
A_{\text {eff }}(t)=A+c_{1} V
$$

where $c_{1}\left[\mathrm{~m}^{2} / \mathrm{V}\right]$ is a parameter that has yet to be determined.

The model assumes two components for the decay of the ion density after current zero. The first is a natural decay, assumed exponential, and the second is from the postarc current. In this way, the ion density is defined as

$$
\frac{d n_{\mathrm{i}}}{d t}=-\frac{n_{\mathrm{i}}}{\tau}-\frac{i_{\mathrm{pa}}}{q_{\mathrm{i}} c_{2}}
$$

where $\tau$ is the time constant for the natural decay, and $c_{2}\left[\mathrm{~m}^{3}\right]$ is a parameter. $i_{\mathrm{pa}}$ is considered to be positive only in (8) to ensure an ion density decay.

We use a sheath capacitance for modeling the displacement current that results from the time change of the electric field inside the sheath. The expression for the sheath capacitance is

$$
C_{\mathrm{sh}}=\frac{K}{V^{3 / 4}}
$$

Appendix describes how we come to (9).

By combining (2), (6), (7), and (9), we arrive at the expression for the postarc current as

$$
i_{\mathrm{pa}}=q_{\mathrm{i}} n_{\mathrm{i}} v_{\mathrm{B}} A_{\mathrm{eff}} \tanh \left(\frac{q_{\mathrm{i}} V}{2 k T_{\mathrm{e}}}\right)+C_{\mathrm{sh}} \frac{d V}{d t} .
$$

When $m_{\mathrm{i}}$ [from $\left.v_{\mathrm{B}},(1)\right], q_{\mathrm{i}}, T_{\mathrm{e}}$, and $A$ are considered constant, the model requires four parameters: $n_{\mathrm{i}}(0), \tau, c_{1}$, and $c_{2}$.

The model has been programmed as a function block in Matlab's SimPowerSystems. For the simulation of the breaker in an electrical circuit, we used the ode23tb solver with an absolute and relative error of $1 \cdot 10^{-5}$. Fig. 6 shows the result of the simulation of the measurement in Fig. 2. We use the same 

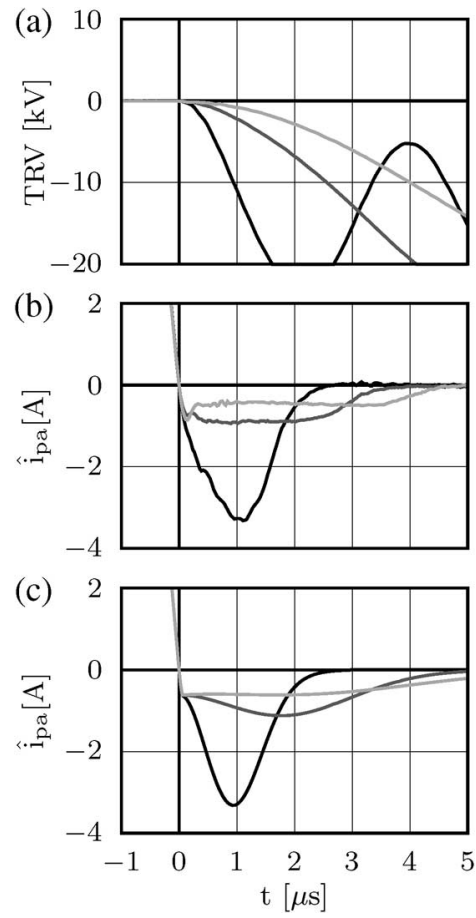

Fig. 7. Simulation of three measurements performed with equal conditions but different TRV. (a) Measured TRV. (b) Measured postarc current. (c) Simulated postarc current.

values for the parameters as in the simulation in Section III, and $c_{1}$ and $c_{2}$ are $0.5 \cdot 10^{-6} \mathrm{~m}^{2} / \mathrm{V}$ and $56 \cdot 10^{-6} \mathrm{~m}^{3}$, respectively.

In the final example, the model simulates three measurements that were performed with equal test settings (same arcing time and same peak short-circuit current) but with different TRVs. Fig. 7 shows the results.

Because the three measurements were performed with equal settings, it should be expected that the plasma conditions at $t=0$ were equal as well. As a consequence, the model's parameters should be the same in all three simulations. The parameters $n_{\mathrm{i}}(0), \tau$, and $c_{2}$ were indeed equal $\left(7 \cdot 10^{17} \mathrm{~m}^{-3}\right.$, $5 \mu \mathrm{s}$, and $33 \cdot 10^{-6} \mathrm{~m}^{3}$, respectively), but only $c_{1}$ had to be adjusted. This value was $2 \cdot 10^{-6} \mathrm{~m}^{2} / \mathrm{V}$ for the measurement with the steepest TRV rise, $1 \cdot 10^{-6} \mathrm{~m}^{2} / \mathrm{V}$ for the middle, and $0.5 \cdot 10^{-6} \mathrm{~m}^{2} / \mathrm{V}$ for the slowest TRV rise. Apparently, our assumed relation between the effective cathode area and the voltage in (7) is inadequate.

\section{Discussion}

The model presented in Section IV simulates the measurements well. Nevertheless, some considerations have to be made about its assumptions and its results.

\section{A. Initial Ion Density and Its Distribution}

We started in Section III with the assumption of an initially stationary plasma, which is equally distributed along the gap. Measurements and simulation on the behavior of ions in a vacuum arc, which are found in the literature, indicate that this assumption is not valid. First, cathode spots launch ions
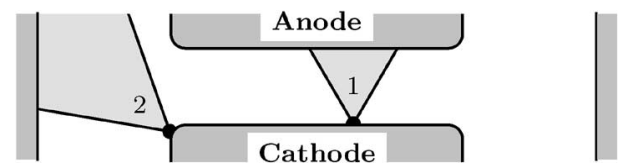

Fig. 8. When the final cathode spot extinguishes at position 1, more charge returns to the electric circuit than when it extinguishes at position 2.

into the plasma with velocities that are much higher than their corresponding thermal energy, and hence, instead of being stationary, ions drift toward the anode. Second, the plasma rarefies as it moves away from the cathode, and finally, additional processes introduce secondary charge inside the gap, which is not generated by a cathode spot. Such processes include the ionization of metal-vapor present inside the gap, which originates, for example, from the anode, which is heated by the bombardment of ions.

With the increase of metal-vapor arising from the anode, more ions that are launched from cathode spots collide with other particles inside the gap, hence reducing their velocity. Therefore, it could be argued that the ion velocity in the immediate proximity of the anode is determined by the ion's thermal velocity only. This justifies the assumption of a stationary plasma for the modeling of at least the low-voltage period, since in this region, the model mainly applies to the thin sheath in front of the postarc cathode.

\section{B. Increase in the Postarc Cathode's Effective Size}

Equation (6) is valid only for a double Langmuir probe with equal probe size, but with (7), we deliberately change the size of one electrode. However, by the time that this affects (6), any other function than this would have also reached the ion saturation current, and hence, we left this equation in its current form.

\section{Sheath Expansion}

The model can be applied for an indefinite time after current zero, because eventually, the ion density $n_{\mathrm{i}}$ will be depleted, after which the postarc current remains zero. However, as the sheath grows in front of the postarc cathode, the geometry of the breaker's inside and the plasma properties should be considered while calculating the postarc current.

\section{Simulated Results Compared With Measured Data}

In Section V-A, some processes are described that determine the plasma density distribution at current zero. However, the final cathode spot's last position has also a strong influence on the postarc plasma properties. When this position is near the edge of the cathode, a significant amount of charge is ejected away from the contacts and disappears, e.g., by recombination at the breaker's vapor shield. If this is the case, less charge is returned to the electrical circuit by means of a postarc current, compared to the situation in which the final cathode spot's position is near the center of the cathode (see Fig. 8). 


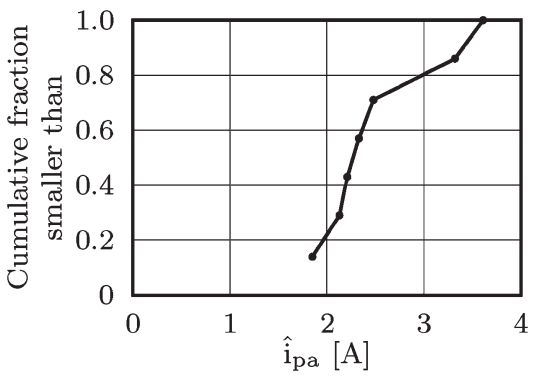

Fig. 9. Cumulative distribution of the measured postarc current's peak value after identical tests. The scatter is largely the result of the cathode spot's final position. The results have been obtained in the short-line fault simulation circuit described in [14] and [18].

Since a cathode spot moves randomly across the cathode (but biased by an external magnetic field), its final position is unknown. As a result, the postarc plasma conditions are different in each measurement, which gives the postarc current a significant random component. This is clearly demonstrated in Fig. 9.

The postarc current's scatter makes it difficult to relate the postarc properties with the arcing conditions. Therefore, integrating more postarc processes in the model would not automatically lead to better results.

\section{E. Model Parameters}

All the parameters used in the model are estimated rather than measured and have been adjusted to make the simulated results fit the measured data. Although those parameters were kept in the range of values found in the literature, it would be better to actually measure them during a test (if possible). In addition, the model becomes more plausible when its parameters $n_{\mathrm{i}}(0), \tau, c_{1}$, and $c_{2}$ are linked to actual physical processes.

\section{CONCLUSION}

The most widely used model for simulating the VCB's interaction with an electrical circuit (Andrews and Varey) lacks a sound explanation for the voltage-zero period of up to $0.5 \mu$ s that immediately follows current interruption. This event has been predicted and measured and strongly resembles the electrical behavior of a double-Langmuir probe. When this probe's theory is applied to the VCB, its result matches the measured data well. Hence, we developed a new model starting from this theory.

Contrary to the original models, the new model takes into account the thermal velocity of charged particles instead of their drift velocity. Measured data found in the literature on thermal and drift velocities in VCBs after breaking high short-circuit currents justify this assumption for at least the initial phase of the postarc current.

Since we are mainly interested in the interaction between the breaker and the electrical circuit, the complicated physics of sheath expansion have been modeled with deliberate pragmatic assumptions. These assumptions involve the following.

1) An exponential function simulates the ion density's natural decay.
2) The postarc current accelerates the ion density's decay.

3) The cathode's effective area expands linearly with the TRV to simulate the cathodic sheath growth.

4) The displacement current, resulting from the time change of the electric field inside the cathode sheath, is simulated with the aid of a voltage-dependent sheath capacitance.

The model requires four parameters for the simulation of a postarc current. These are the ion density at current zero, the time constant for the ion density's natural decay, and two arbitrary constants, one relating the postarc current to the accelerated ion density decay and another to relate the TRV to the cathode's effective area increment.

After fitting the model's parameters, its results match the measured data well. The model is less complicated to implement; it requires less computation time, and it is more stable than the previous postarc current model. Therefore, it is a suitable alternative for modeling the breaker's postarc interaction with an electrical circuit.

\section{APPENDIX \\ Determination of The Sheath Capacitance}

For the evaluation of the sheath capacitance, we follow the method used in [22, p. 440]. In the case that the ion energy at the plasma-sheath edge is small compared to the applied potential, the ion energy and flux conservation equations can be written as

$$
\begin{aligned}
\frac{1}{2} m_{\mathrm{i}} v_{\mathrm{i}}^{2}(x) & =-q_{\mathrm{i}} \phi(x) \\
q_{\mathrm{i}} n_{\mathrm{i}}(x) v_{\mathrm{i}}(x) & =J_{0}
\end{aligned}
$$

where $v_{\mathrm{i}}(x)$ is the ion velocity inside the sheath, $\phi(x)$ is the potential (defined 0 at the plasma-sheath edge and $-V$ at the cathode), and $J_{0}$ is the constant ion current density. Solving (11) and (12) for $n_{\mathrm{i}}(x)$, we obtain

$$
n_{\mathrm{i}}(x)=\frac{J_{0}}{q_{\mathrm{i}}}\left(-\frac{2 q_{\mathrm{i}} \phi}{m_{\mathrm{i}}}\right)^{-1 / 2}
$$

Using this in Poission's equation $\left(d^{2} \phi / d x^{2}=-q_{\mathrm{i}} n_{\mathrm{i}}(x) / \epsilon_{0}\right)$, we find

$$
\frac{d^{2} \phi}{d x^{2}}=-\frac{J_{0}}{\epsilon_{0}}\left(-\frac{2 q_{\mathrm{i}} \phi}{m_{\mathrm{i}}}\right)^{-1 / 2}
$$

where $\epsilon_{0}$ is the permittivity in vacuum. Multiplying (14) with $d \phi / d x$ and integrating from the plasma-sheath edge to the cathode, we have

$$
\frac{1}{2}\left(\frac{d \phi}{d x}\right)^{2}=2 \frac{J_{0}}{\epsilon_{0}}\left(\frac{2 q_{\mathrm{i}}}{m_{\mathrm{i}}}\right)^{-1 / 2}(-\phi)^{1 / 2}
$$

where we assume $d \phi / d x=-E=0$ at the plasma-sheath boundary. 
The sheath capacitance is written as

$$
C_{\mathrm{sh}}=\frac{d Q}{d V}=\epsilon_{0} A_{\mathrm{eff}} \frac{d E}{d V}
$$

where $Q$ is the charge on the cathode, $V$ is the sheath voltage, $A_{\text {eff }}$ is the effective cathode area, as defined in (7), and $E$ is the electric field at the cathode. Equation (15) is rewritten as

$$
E=2\left(\frac{J_{0}}{\epsilon_{0}}\right)^{1 / 2}\left(\frac{m_{\mathrm{i}} V}{2 q_{\mathrm{i}}}\right)^{1 / 4}
$$

then differentiated with respect to $V$ and used in (16), to find the sheath capacitance as

$$
C_{\mathrm{sh}}=\frac{1}{2}\left(\epsilon_{0} J_{0}\right)^{1 / 2}\left(\frac{m_{\mathrm{i}}}{2 q_{\mathrm{i}}}\right)^{1 / 4} A_{\mathrm{eff}} V^{-3 / 4} .
$$

The current density is equal throughout the sheath, and since we assume that ions enter the sheath at Bohm velocity (1), we know $J_{0}$ from (12), and (18) can be rewritten as

$$
C_{\mathrm{sh}}=\frac{K}{V^{3 / 4}}
$$

where

$$
K \approx 0.42\left(q_{\mathrm{i}} n_{\mathrm{i}} \epsilon_{0}\right)^{1 / 2}\left(\frac{k T_{\mathrm{e}}}{q_{\mathrm{i}}}\right)^{1 / 4} A_{\mathrm{eff}} .
$$

\section{REFERENCES}

[1] L. van der Sluis, Transients in Power Systems. New York: Wiley, 2000.

[2] S. Stewart, Distribution Switchgear. London, U.K.: IEE, 2004.

[3] A. Greenwood, Vacuum Switchgear. London, U.K.: IEE, 1994.

[4] M. Reece, "The vacuum switch, Part 2. Extinction of an a.c. vacuum arc," Proc. Inst. Electr. Eng., vol. 110, no. 4, pp. 803-811, 1963.

[5] S. Yanabu, M. Homma, E. Kaneko, and T. Tamagawa, "Post arc current of vacuum interrupters," IEEE Trans. Power App. Syst., vol. PAS-104, no. 1, pp. 166-172, Jan. 1985.

[6] E. Dullni, E. Schade, and B. Gellert, "Dielectric recovery of vacuum arcs after strong anode spot activity," in Proc. XIIth ISDEIV, Shoresh, Israel, 1986, pp. 538-544.

[7] G. Lins, "Influence of electrode separation on ion density in the vacuum arc," IEEE Trans. Plasma Sci., vol. 19, no. 5, pp. 718-724, Oct. 1991.

[8] K. Steinke, M. Lindmayer, and K.-D. Weltmann, "Post-arc currents of vacuum interrupters with axial magnetic field contacts under high current and voltage stress," in Proc. XIXth ISDEIV, Xi' an, China, 2000, pp. $475-480$.

[9] G. Düning and M. Lindmayer, "Energy and density of ions in vacuum arcs between axial and radial magnetic field contacts," IEEE Trans. Plasma Sci., vol. 29, no. 5, pp. 726-733, Oct. 2001.

[10] S. Childs and A. Greenwood, "A model for dc interruption in diffuse vacuum arcs," IEEE Trans. Plasma Sci., vol. PS-8, no. 4, pp. 289-294, 1980.

[11] M. Glinkowski and A. Greenwood, "Computer simulation of post-arc plasma behavior at short contact separation in vacuum," IEEE Trans. Plasma Sci., vol. 17, no. 1, pp. 45-50, Feb. 1989.

[12] M. T. Glinkowski and P. Stoving, "Numerical modeling of vacuum arc interruption based on the simplified plasma equations," IEEE Trans. Magn., vol. 31, no. 3, pp. 1924-1927, May 1995.

[13] J. G. Andrews and R. H. Varey, "Sheath growth in a low pressure plasma," Phys. Fluids, vol. 14, no. 2, pp. 339-343, Feb. 1971.

[14] E. van Lanen, M. Popov, L. van der Sluis, R. Smeets, and R. Kerkenaar, "VCB current zero phenomena modelled for circuit analysis," in Proc. 21st ISDEIV, Yalta, Ukraine, 2004, pp. 418-421.

[15] S. Baboolal, "Boundary conditions and numerical fluid modelling of timeevolutionary plasma sheaths," J. Phys. D, Appl. Phys., vol. 35, no. 7, pp. 658-664, Apr . 2002.
[16] S. W. Rowe and J. P. Boeuf, "Simulation of plasma sheath dynamics in vacuum circuit breakers," in Proc. 20th ISDEIV, 2002, pp. 162-165.

[17] M. Keidar and I. I. Beilis, "Transition from plasma to space-charge sheath near the electrode in electrical discharges," IEEE Trans. Plasma Sci. vol. 33, no. 5, pp. 1481-1486, Oct. 2005.

[18] R. P. P. Smeets and W. A. van der Linden, "Current-zero measurements of vacuum circuit breakers interrupting short-line faults," IEEE Trans. Plasma Sci., vol. 31, no. 5, pp. 852-858, Oct. 2003.

[19] E. van Lanen, M. Popov, L. van der Sluis, and R. Smeets, "Vacuum circuit breaker current-zero phenomena," IEEE Trans. Plasma Sci., vol. 33, no. 5, pp. 1589-1593, Oct. 2005.

[20] R. Gebel, "Vapor shield potential in vacuum interrupters after clearing of short circuits," IEEE Trans. Plasma Sci., vol. 17, no. 5, pp. 834-838, Oct. 1989.

[21] Y. P. Raizer, Gas Discharge Physics, J. E. Allen, Ed. Berlin, Germany: Springer-Verlag, 1991.

[22] M. A. Lieberman and A. J. Lichtenberg, Principles of Plasma Discharges and Materials Processing. Hoboken, NJ: Wiley, 2005.

[23] R. L. Boxman, D. M. Sanders, and P. J. Martin, Handbook of Vacuum Arc Science and Technology; Fundamentals and Applications. Park Ridge, NJ: Noyes, 1995.

[24] M. Galonska, R. Hollinger, I. A. Krinberg, and P. Spaedtke, "Influence of an axial magnetic field on the electron temperature in a vacuum arc plasma," IEEE Trans. Plasma Sci., vol. 33, no. 5, pp. 1542-1547, Oct. 2005.

[25] S. Nam, B. Lee, S. Park, S. Kim, and Y. Han, "Spectroscopic measurement of high current vacuum arc plasma in triggered vacuum switch," in Proc. PPPS, Jun. 2001, vol. 2, pp. 1790-1793.

[26] K. Arai, S. Takahashi, O. Morimiya, and Y. Niwa, "Probe measurements of residual plasma of a magnetically confined high-current vacuum arc," IEEE Trans. Plasma Sci., vol. 31, no. 5, pp. 929-933, Oct. 2003.

[27] A. Klajn, "Langmuir probes in switching vacuum arc measurements," IEEE Trans. Plasma Sci., vol. 33, no. 5, pp. 1611-1617, Oct. 2005.

[28] A. Klajn, "Experimental analysis of ion parameters during the forced vacuum arc interruption," in Proc. 20th ISDEIV, Tours, France, 2002, pp. 415-418.

[29] H. Pursch and B. Jüttner, "The behaviour of the ion current at the extinction of high current vacuum arcs," in Proc. XVIIth ISDEIV, Berkeley, CA, 1996, pp. $169-172$

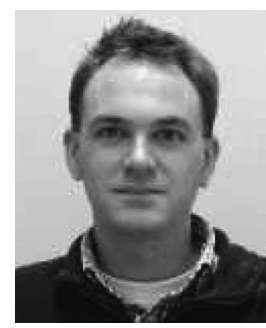

Ezra P. A. van Lanen (M'03) was born in Eindhoven, The Netherlands, on February 6, 1975. He received the M.Sc degree in electrical engineering from Delft University of Technology, Delft, The Netherlands, in 2002, where he is currently working toward the Ph.D. degree on digital testing of vacuum circuit breakers.

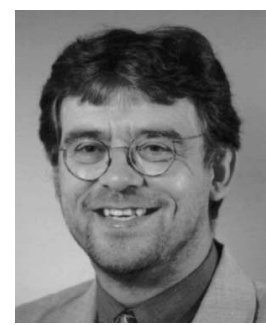

René Peter Paul Smeets (SM'02) received the M.Sc. degree in physics, in 1981, and the Ph.D. degree for research work on vacuum arcs both from the Eindhoven University of Technology, Eindhoven, The Netherlands.

During 1991, he worked with Toshiba Corporation's Heavy Apparatus Engineering Laboratory, Japan. Until 1995, he was an Assistant Professor at the Eindhoven University. In 1995, he joined KEMA T\&D Testing Services, Arnhem, The Netherlands, where he currently manages the R\&D activities of KEMA's High Power Laboratory. In 2001, he was appointed Parttime Professor at the Eindhoven University of Technology. He published over 130 papers on high-power switching and testing in international magazines and conference proceedings.

Dr. Smeets was Guest Editor for the IEEE TRANSACTIONS ON Plasma SCIENCE Special Issue on Vacuum Discharge Plasmas, in 1999. He has been Chairman and member of various International Electrical Congresses and the International Council on Large Electric Systems study groups. He is Chairman of the Vacuum Circle of the Current Zero Club. 


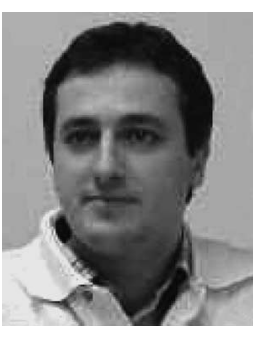

Marjan Popov (M'95-SM'03) received the Dipl.Ing. and M.S. degrees in electrical engineering from Sts. Cyril and Methodius University, Skopje, Republic of Macedonia, in 1993 and 1998, respectively, and received the Ph.D. degree from Delft University of Technology, Delft, The Netherlands, in 2002.

From 1993 to 1998, he was a Teaching and Research Assistant in the Faculty of Electrical Engineering, University of Skopje, spending 1997 as a Visiting Researcher at the University of Liverpool, Liverpool, U.K. He is currently Assistant Professor in the Electrical Power Systems Group, Power Systems Laboratory, Delft University of Technology. His research interests are in arc modeling, transients in power systems, parameter estimation, and relay protection.

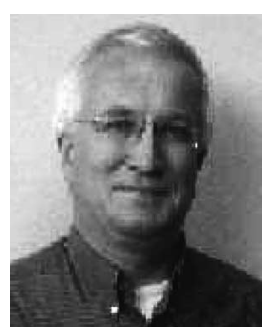

Lou van der Sluis (M'82-SM'86) was born in Geervliet, The Netherlands, on July 10, 1950. He received the M.Sc. degree in electrical engineering from Delft University of Technology, Delft, The Netherlands, in 1974

He was with the KEMA High Power Laboratory in 1977 as a Test Engineer and was involved in the development of a data acquisition system for the High Power Laboratory, computer calculations of test circuits, and the analysis of test data by digital computer. In 1990, he became a Part-time Professor, and since 1992, he has been employed as a Full-time Professor in Power Systems Department, Delft University of Technology.

Prof. van der Sluis is a Past Chairman of CC-03 of Cigre and Cired to study the transient recovery voltages in medium and high voltage networks. 